\title{
The degree of $\mathrm{CO}$ depletion in pre-stellar cores ${ }^{\star}$
}

\author{
A. Bacmann ${ }^{1, \star \star}$, B. Lefloch ${ }^{2}$, C. Ceccarelli ${ }^{3,2}$, A. Castets $^{3}$, J. Steinacker ${ }^{1}$, and L. Loinard ${ }^{4}$ \\ 1 Astrophysikalisches Institut und Universitäts Sternwarte (AIU), Schillergäßchen 2-3, 07745 Jena, Germany \\ e-mail: stein@astro.uni-jena.de \\ 2 Laboratoire d'Astrophysique, Observatoire de Grenoble, BP 53, 38041 Grenoble Cedex 9, France \\ e-mail: lefloch@obs.ujf-grenoble.fr \\ 3 Observatoire de Bordeaux, 2 rue de l'Observatoire, BP 89, 33270 Floirac, France \\ e-mail: ceccarel@observ.u-bordeaux.fr, castets@observ.u-bordeaux.fr \\ 4 Instituto de Astronomía, Universidad Nacional Autónoma de México, Apartado Postal 3-72 (Xangari), \\ 58089 Morelia, Michoacán, Mexico \\ e-mail: 1. loinard@astrosmo.unam.mx
}

Received 5 March 2002 / Accepted 24 April 2002

\begin{abstract}
We present new results on CO depletion in a sample of nearby pre-stellar cores, based on observations of the millimeter $\mathrm{C}^{17} \mathrm{O}$ and $\mathrm{C}^{18} \mathrm{O}$ lines and the $1.3 \mathrm{~mm}$ dust emission with the IRAM $30 \mathrm{~m}$ telescope. In most cases, the distribution of $\mathrm{CO}$ is much flatter than that of the dust, whereas other tracers, like $\mathrm{N}_{2} \mathrm{H}^{+}$, still probe the latter. In the centre of these objects, we estimate CO to be underabundant by a factor 4-15 depending on the cores. The $\mathrm{CO}$ underabundance is more pronounced in the central regions and appears to decrease with increasing distance from the core centre. This underabundance is most likely due to the freezing out of CO onto the dust grains in the cold, dense parts of the cores. We find evidence for an increase of the CO depletion degree with the core density.
\end{abstract}

Key words. ISM: molecules - dust, extinction - stars: formation

\section{Introduction}

Of all the molecules present in the interstellar medium, $\mathrm{CO}$ (and its isotopes) has been the object of particular attention. It has been widely used to study the mass distribution and kinematics of star forming molecular clouds. Moreover, CO is thought to play a major role in the thermal cooling of molecular clouds (e.g., Goldsmith \& Langer 1978). In very dense and cold regions of the interstellar medium the $\mathrm{CO}$ molecules tend to leave the gas phase and condense out onto the dust grains, directly affecting the thermal balance of clouds (Goldsmith 2001). CO also participates in a complex chemical network and its depletion leads to changes in gas composition and chemical reactions. These, in turn, can influence the contraction of molecular clouds through ambipolar diffusion (e.g. Shu et al. 1997), via the ionisation fraction (Caselli et al. 1998 ) or the gas phase deuteration of molecules (Roberts \& Millar 2000a, 2000b).

This phenomenon affects in particular pre-stellar condensations, which are characterized by very low temperatures $(\sim 10 \mathrm{~K})$ and high central densities

Send offprint requests to: A. Bacmann,

e-mail: bacmann@astro.uni-jena.de

* Based on observations collected at the IRAM 30 m, Spain.

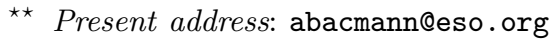

$\left(\sim 10^{4}-10^{5} \mathrm{~cm}^{-3}\right)$. These objects, still starless, are thought to evolve slowly via ambipolar diffusion towards higher degrees of condensation until they become unstable and collapse under their own gravity to form a protostar. This pre-collapse phase is believed to strongly influence the following stages of the star formation process - mass infall rate (e.g. Henriksen et al. 1997), fragmentation, mass of future star (e.g. Bacmann et al. 2000). It is therefore of prime importance to characterize the physical and chemical conditions in the gas and dust of pre-stellar condensations which have not yet started to collapse. The study of CO depletion is in this context particularly relevant due to the implications of e.g. modified thermal balance or contraction by ambipolar diffusion induced by a decreased abundance of the molecule. CO depletion in dense cores has already been discussed by Kramer et al. (1999), Willacy et al. (1998), and Caselli et al. (1999, 2002a, 2002b) who focussed on L1544, a core similar to the ones we discuss in this work. Here, we studied the distribution and abundance of $\mathrm{CO}$ via its rarer isotopes $\mathrm{C}^{17} \mathrm{O}(1-0)$ and $\mathrm{C}^{18} \mathrm{O}(1-0)$, and of $\mathrm{N}_{2} \mathrm{H}^{+}(1-0)$ in a sample of pre-stellar cores, using the $1.3 \mathrm{~mm}$ continuum as a tracer of the $\mathrm{H}_{2}$ column density. Our aim was to quantify the depletion and to link it with physical properties of the cores. We first briefly present the observations in Sect. 2, then the spatial distributions and 
column density estimations in Sect. 3 , discuss our results and conclude in Sect. 4.

\section{Observations and data reduction}

We have selected a sample of 7 pre-stellar cores previously studied by Bacmann et al. (2000, cf. Table 1$)$. The core L1544, studied in detail by Caselli et al. (1999, 2002a, 2002b), has also been included for comparison.

Observations in the millimeter lines of $\mathrm{N}_{2} \mathrm{H}^{+}(1-$ $0), \quad \mathrm{C}^{17} \mathrm{O}(1-0), \quad \mathrm{C}^{18} \mathrm{O}(2-1)$ and $(1-0)$ were made in August 2000 and 2001 at the IRAM $30 \mathrm{~m}$ telescope. The spectra were taken in position switching mode with a $20^{\prime \prime}$ sampling along cuts in chosen directions passing through the maximum of the $1.3 \mathrm{~mm}$ emission. The reference positions were checked to be free of $\mathrm{C}^{18} \mathrm{O}(1-0)$ emission. We used an autocorrelator with a resolution of $20 \mathrm{kHz}$ as backend. The spectral resolution was degraded so as to provide a kinematic resolution of $\sim 0.1 \mathrm{~km} \mathrm{~s}^{-1}$ at all wavelengths. Pointing was checked typically every 1.5 hour and found to be better than $\sim 3^{\prime \prime}$. The data were reduced using the CLASS package. The line fluxes are expressed in units of main-beam brightness temperatures. The uncertainties in the fluxes are $\approx 10 \%$. The beam size $\left(\sim 22^{\prime \prime}\right.$ at $\left.110 \mathrm{GHz}\right)$ and the efficiencies (FWHM) are taken from the IRAM web page http://www.iram.es/. Previous mapping of the cores in the 1.3 millimeter continuum is described in Bacmann et al. (2000).

\section{Results}

\subsection{Distribution of molecular and dust tracers}

We compared the spatial distribution of $\mathrm{CO}, \mathrm{N}_{2} \mathrm{H}^{+}$and of the dust traced by the $1.3 \mathrm{~mm}$ continuum emission. To this effect, we plotted for each core the integrated intensity of the molecular lines $\left(\mathrm{C}^{18} \mathrm{O}(1-0), \mathrm{C}^{17} \mathrm{O}(1-0)\right.$ and $\left.\mathrm{N}_{2} \mathrm{H}^{+}(1-0)\right)$ together with the millimeter continuum flux, along directions passing through (or close to) the maximum of the $1.3 \mathrm{~mm}$ continuum map. To obtain the integrated line intensities, we first fitted a Gaussian (or a series of Gaussians in the case of hyperfine structures) to the considered line, and then evaluated the area under the Gaussian. The results obtained are similar if we consider the integrated signal between two given velocities. The resolution of the $1.3 \mathrm{~mm}$ continuum map was degraded to the resolution of the molecular line observations, $22^{\prime \prime}$. The resulting plots are presented in Fig. 1 for all cores except L1544. Whereas the continuum is sharply peaked (except for L1709A), the distribution of the $\mathrm{C}^{18} \mathrm{O}(1-0)$ and $\mathrm{C}^{17} \mathrm{O}(1-0)$ integrated intensities is flatter (e.g. L1689B and L310) or completely fails to trace the strong continuum peak (e.g. L429 and Oph D). The core L328 is particular in the sense that the CO isotopes are also sharply peaked (their distribution is only slightly broader than that of the dust: $\sim 15 \%$ broader at half-maximum). For all cores, the distribution of $\mathrm{N}_{2} \mathrm{H}^{+}$, on the other hand, closely follows that of the dust traced by the continuum.
These results tend to show that CO is underabundant with respects to $\mathrm{H}_{2}$ (traced by the continuum) in the centres of the cores. As Caselli et al. (1999) pointed out, this is most likely due to the depletion of $\mathrm{CO}$ onto the dust grains, because of the high densities and low temperatures in the inner parts of the cores. However, it can already be seen from the various behaviours of the plots such as those in Fig. 1 that the amplitude of this phenomenon, the depletion degree, is not the same in all the cores considered.

\subsection{Physical conditions}

The average $\mathrm{H}_{2}$ density at the emission peak in the cores was estimated to be of a few $10^{5} \mathrm{~cm}^{-3}$ in the $11^{\prime \prime}$ telescope beam by Bacmann et al. (2000, see also Table 1$)$. Hence, the populations of the energy levels of CO and its isotopes are very close to thermalization. The ratio of the $\mathrm{C}^{17} \mathrm{O}(2-1)$ to $(1-0)$ line integrated areas, calculated under the assumptions of optically thin lines and at LTE, therefore provides a good estimate of the kinetic temperature. We applied the same procedure to the $\mathrm{C}^{18} \mathrm{O}$ lines under the same assumptions. We obtained consistent results between both isotopes, with values in the range 6 $8 \mathrm{~K}$ for the $\mathrm{C}^{17} \mathrm{O}$ lines and in the range $7.5-10 \mathrm{~K}$ for $\mathrm{C}^{18} \mathrm{O}$. The results are summarized in Table 1 . Such temperatures are similar to those found towards other pre-stellar and/or protostellar cores (Caselli et al. 1999; Fuller \& Myers 1993; Ladd et al. 1998).

We evaluated the CO column density using the rare isotope $\mathrm{C}^{17} \mathrm{O}$ in order to make sure our results were not affected by opacity effects. The opacity of the $\mathrm{C}^{18} \mathrm{O}(1-0)$ transition was determined following the method described by Ladd et al. (1998). Supposing a $\left[{ }^{17} \mathrm{O}\right] /\left[{ }^{18} \mathrm{O}\right]$ abundance ratio of 3.65 (Wilson \& Rood 1994; Penzias 1981), we derived opacities lower than 0.1 for Oph D, L328 and L310, of 1.1 for L429, 1.3 and 1.5 for L1709A and L1689B, respectively. According to the aforementioned $\mathrm{C}^{18} \mathrm{O}(1-0)$ opacities, the $\mathrm{C}^{17} \mathrm{O}(1-0)$ transition is optically thin for all of the cores. This validates our determination of the kinetic temperature from the $\mathrm{C}^{17} \mathrm{O}$ lines. Although the opacity effects are small in the general case, we prefered to use the same tracer $\mathrm{C}^{17} \mathrm{O}$ for all cores for more accurate comparisons. The $\mathrm{C}^{17} \mathrm{O}$ column density was determined under the same hypothesis and adopting as kinetic temperature the values derived previously. The values of $N_{\mathrm{C}^{17} \mathrm{O}}$ determined at the continuum emission peak are presented in Table 1.

The $\mathrm{H}_{2}$ column density was determined from the dust thermal emission at $1.3 \mathrm{~mm}$, which is largely optically thin. We degraded the resolution of all our continuum maps to the resolution at the frequency of the $\mathrm{C}^{17} \mathrm{O}(1-$ 0 ) line, so as to compare $\mathrm{H}_{2}$ and $\mathrm{C}^{17} \mathrm{O}$ column densities in the same beam. The dust temperature was taken to be $7.5 \mathrm{~K}$ in all cores at their centre, as suggested by the recent works of Zucconi et al. (2001) and Evans et al. (2001). This is slightly lower than in the envelope, where values of $11 \mathrm{~K}$ were determined from ISOPHOT 
Table 1. Comparison of $\mathrm{C}^{17} \mathrm{O}$ and (calculated from dust) $\mathrm{H}_{2}$ properties. The $\mathrm{H}_{2}$ densities are taken from Table 3 of Bacmann et al. (2000). Errors on the temperature are typically $\pm 1 \mathrm{~K}$. Uncertainties on $N_{\mathrm{H}_{2}}$ and $n\left(\mathrm{H}_{2}\right)$ are a factor of $\sim 2$, those on $X$ and $f$ a factor of $\sim 2.5$.

\begin{tabular}{|c|c|c|c|c|c|c|c|c|c|}
\hline Name & $\begin{array}{c}\int T_{\mathrm{mb}}^{10}\left(\mathrm{C}^{17} \mathrm{O}\right) \mathrm{d} v \\
\mathrm{~K} \mathrm{~km} \mathrm{~s}\end{array}$ & $\begin{array}{l}T_{\mathrm{k}} \\
(\mathrm{K})\end{array}$ & $\begin{array}{l}N_{\mathrm{C}^{17} \mathrm{O}} \\
\left(\mathrm{cm}^{-2}\right)\end{array}$ & $\begin{array}{c}N_{\mathrm{H}_{2}} \\
\left(\mathrm{~cm}^{-2}\right)\end{array}$ & $\begin{array}{c}n\left(\mathrm{H}_{2}\right) \\
\left(\mathrm{cm}^{-3}\right)\end{array}$ & $\begin{array}{c}N_{\mathrm{C}^{17} \mathrm{O}} / N_{\mathrm{H}_{2}} \\
(=X)\end{array}$ & $\begin{array}{c}f \\
\left(=X_{\mathrm{can}} / X\right)\end{array}$ & $f^{70}$ & $f^{50}$ \\
\hline L1544 & - & 10 & $5.6 \pm 0.8 \times 10^{14}$ & $1.6 \times 10^{23}$ & $4 \times 10^{5}$ & $3.5 \times 10^{-9}$ & 14 & - & - \\
\hline L1689B & $1.42( \pm 0.14)$ & 9.3 & $1.5 \pm 0.2 \times 10^{15}$ & $1.4 \times 10^{23}$ & $1.4 \times 10^{5}$ & $1.1 \times 10^{-8}$ & 4.5 & 2 & 1.5 \\
\hline L1709A & $1.18( \pm 0.12)$ & 10 & $1.2 \pm 0.2 \times 10^{15}$ & $1.4 \times 10^{23}$ & $1.2 \times 10^{5}$ & $8.6 \times 10^{-9}$ & 5.5 & 2.5 & 2 \\
\hline L310 & $0.35( \pm 0.03)$ & 8.8 & $2.8 \pm 0.5 \times 10^{14}$ & $7.3 \times 10^{22}$ & $9 \times 10^{4}$ & $3.8 \times 10^{-9}$ & 12.5 & 5 & 3 \\
\hline L328 & $0.60( \pm 0.06)$ & 9.7 & $5.3 \pm 0.8 \times 10^{14}$ & $9.5 \times 10^{22}$ & $1.8 \times 10^{5}$ & $5.6 \times 10^{-9}$ & 8.5 & 5 & - \\
\hline L429 & $0.50( \pm 0.05)$ & 7.5 & $4.3 \pm 0.8 \times 10^{14}$ & $1.4 \times 10^{23}$ & $6 \times 10^{5}$ & $3.1 \times 10^{-9}$ & 15.5 & 5 & 3 \\
\hline Oph D & $0.57( \pm 0.06)$ & 8.2 & $4.8 \pm 0.9 \times 10^{14}$ & $1.4 \times 10^{23}$ & $3 \times 10^{5}$ & $3.4 \times 10^{-9}$ & 14 & 4.5 & 3 \\
\hline
\end{tabular}

observations (Ward-Thompson et al. 2001). Following André et al. (1996), we have adopted a dust opacity coefficient $\kappa_{1.3 \mathrm{~mm}}=0.005 \mathrm{~cm}^{2} \mathrm{~g}^{-1}$, appropriate for gas in the central pre-stellar regions, where the $\mathrm{H}_{2}$ density is $\sim 10^{5} \mathrm{~cm}^{-3}$, though the actual value is uncertain by a factor of 2 (Preibisch et al. 1993). The values of the $\mathrm{H}_{2}$ column density at the maximum of the $1.3 \mathrm{~mm}$ continuum emission are shown in Table 1.

\section{Discussion}

The results presented above show strong evidence for $\mathrm{CO}$ depletion onto the dust grains in the densest parts of the molecular cloud cores. We present in Table 1 the ratio $X$ of the $\mathrm{C}^{17} \mathrm{O}$ and the $\mathrm{H}_{2}$ column densities. For all cores, the abundance $X$ found is lower than the "canonical" abundance determined by Frerking et al. (1982) towards dark cores $X_{\text {can }}=4.8 \times 10^{-8}$ and varies from 4.5 times smaller to nearly 16 times smaller than $X_{\text {can }}$. The CO molecules thus appear underabundant with respect to $\mathrm{H}_{2}$ at the centre of the pre-stellar cores. The values found suggest different depletion degrees for the different cores, as was already hinted at by the cuts of Fig. 1 . The depletion factor $f$, defined as $X_{\text {can }} / X$, is also shown in Table 1. For L1544, we derive a depletion factor of 14, the difference with the value of 10 found by Caselli et al. (1999) being due to our assumption of a colder central dust temperature. Adopting a higher value for the dust absorption coefficient $\kappa_{1.3 \mathrm{~mm}}$, representative of denser environments would decrease the depletion factor to 6 in Oph D, L429, L310. This case is extremely unlikely as it requires typical densities of $\sim 10^{6} \mathrm{~cm}^{-3}$ over a size of $20^{\prime \prime}$ (3200 AU). The depletion factor would also be a factor of $\sim 2$ smaller if the central temperature is taken to be $11 \mathrm{~K}$ instead of $7.5 \mathrm{~K}$. These results considerably extend the previous works on depletion in pre-stellar cores by Kramer et al. (1999), Caselli et al. (1999) and more recently by Tafalla et al. (2002). The latter report similar depletion factors for L1544 and 4 other pre-stellar cores in the Taurus molecular cloud.

The effect of CO underabundance is still detected at large scale, with a smaller amplitude, in regions covering large parts of the lower density gas, where the "standard" value of $\kappa_{1.3 \mathrm{~mm}}\left(0.005 \mathrm{~cm}^{2} \mathrm{~g}^{-1}\right)$ is a reasonable approximation. For each core, we derived the $\mathrm{C}^{17} \mathrm{O}$ and $\mathrm{H}_{2}$ column density along our cuts at $70 \%$ and $50 \%$ of the maximum of the continuum emission. The values of $70 \%$ and $50 \%$ were chosen as a compromise between having regions of lower density and not being affected by the loss of sensitivity of the millimeter continuum at the edges of the $1.3 \mathrm{~mm}$ maps (e.g. André et al. 1996). We assumed higher dust temperatures of $10 \mathrm{~K}$ and $11 \mathrm{~K}$ to derive the depletion factors $f^{70}$ and $f^{50}$, respectively, since the outer layers are thought to be warmer than the core centre (e.g. Zucconi et al. 2001). The abundance $X^{70}$ is 2 to 5 times smaller than $X_{\text {can }}$, the $C O$ underabundance is more pronounced in the densest, central regions. For all cores, the average depletion factor $f^{70}$ is lower than $f$, and $f^{50}$ is lower than $f^{70}$.

Such variations can be easily understood if depletion is the cause of this underabundance of CO. Indeed, the less dense and warmer parts of the cores should be less affected by the freezing out of $\mathrm{CO}$ molecules onto dust grains. Within the objects, the variations in density are about one order of magnitude, based on the density fits of Tafalla et al. (2002), whereas the temperature varies by $\sim 50 \%$ at most. Hence, the variations of the depletion degree are probably dominated by the variations in density and not in temperature. We stress the fact that the relative variations between $f, f^{70}$ and $f^{50}$ remain true even if the temperature is assumed uniform in the cores. This relation shows that the depletion factor increases with density inside of the core, independently of any modelling of the internal core structure. The increase in $f$ is particularly pronounced in the case of the densest cores L429 and Oph D.

We tried to relate the $\mathrm{CO}$ underabundance with the physical properties of the core. To this effect, we plotted the depletion factor $f$ as a function of the mean density in the core central region (Fig. 2). The above plot indicates an increase of the depletion factor with density, confirming the trend observed for individual cores. A linear regression of the form $f \propto n\left(\mathrm{H}_{2}\right)^{\alpha}$ performed on the whole data set yields $\alpha \simeq 0.1-0.5$. The rather large range of values allowed for the exponent is mainly due to the point L310 which stands out rather markedly of the trend mentionned above. Indeed, leaving aside the 

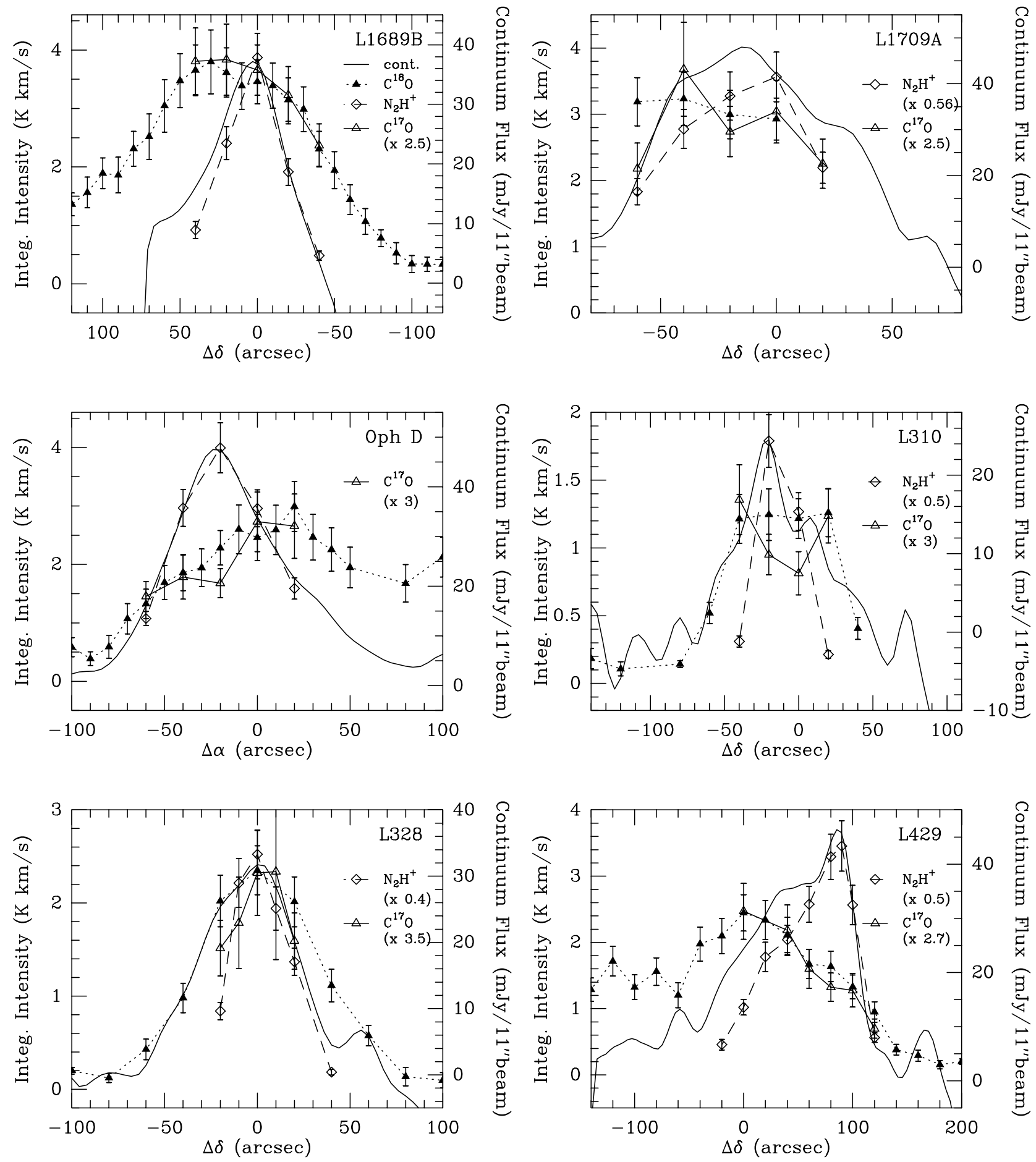

Fig. 1. Spatial distribution of the integrated line intensity of $\mathrm{C}^{18} \mathrm{O}(1-0)$ (filled triangles), $\mathrm{C}^{17} \mathrm{O}(1-0)\left(\right.$ empty triangles), $\mathrm{N}_{2} \mathrm{H}^{+}(1-$ 0 ) (empty diamonds), and of the dust continuum emission (solid line) at $1.3 \mathrm{~mm}$, along cuts in RA, Dec, or the diagonal $\Delta \alpha=\Delta \delta$ (for L310 and L1709A) passing through the maximum of the continuum emission.

L310 data, the points match rather well a relation of the form $f \propto n\left(\mathrm{H}_{2}\right)^{0.7-0.8}$. We note that the observational uncertainties are much larger on L310 than on the rest of the dataset. First, the weather was noticeably unstable when L310 was observed. Because the core is highly elliptical, the pointing fluctations induced by anomalous refraction could lead to underestimating the flux of the $\mathrm{C}^{17} \mathrm{O}(1-0)$ line. Second, in deriving the average density in L310, Bacmann et al. (2000) neglected the high eccentricity of the core. They took as size scale the dimension along its major axis, which leads to an underestimate in their derivation of the density. The L310 point should be actually shifted in the direction of a lower depletion degree and a higher density, as indicated by the arrow in the graph, so that the L310 point probably lies closer to the rest of the data. Therefore, the depletion degree appears 


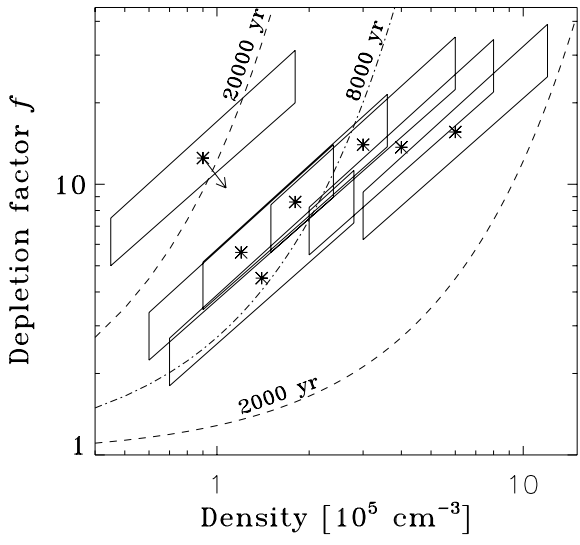

Fig. 2. Variations of the depletion factor $f=X_{\text {can }} / X$ as a function of the mean central density. The parallellograms represent the error boxes for each point, accounting for the fact that the error on $\kappa_{1.3 \mathrm{~mm}}$ affects both density and depletion estimates simultaneously. The arrow indicates the direction the point for L310 would move with better density estimates and more reliable $\mathrm{C}^{17} \mathrm{O}$ measurements. The dashed and dash-dotted lines represent the simple model $f=\exp \left(t / \tau_{\mathrm{d}}\right)$, where $\tau_{\mathrm{d}}$ is the depletion timescale, for various core ages.

to increase with density, following a power law close to $f \propto n\left(\mathrm{H}_{2}\right)^{0.4-0.8}$.

However, one has to be cautious about the "exact" power law coefficient, given the uncertainties in the $\mathrm{H}_{2}$ density, due for the most part to the uncertainty in $\kappa_{1.3 \mathrm{~mm}}$. The above relation was derived assuming a constant dust opacity coefficient and one may wonder how the exponent would be affected in the case $\kappa_{1.3 \mathrm{~mm}}$ increases with density. In such a case, the $\mathrm{H}_{2}$ column density differs from the evaluation by Bacmann et al. (2000), which, in turns, affects the estimation of the depletion degree too. We adopt a power law $\kappa_{1.3 \mathrm{~mm}} \propto n\left(\mathrm{H}_{2}\right)^{\beta}$, where $\beta \sim 0.2$ under the standard assumption that $\kappa_{1.3 \mathrm{~mm}}$ increases by a factor of 2 (from 0.005 to $0.01 \mathrm{~cm}^{2} \mathrm{~g}^{-1}$ ) when the density increases from $10^{5}$ to $10^{6} \mathrm{~cm}^{-3}$ (Preibisch et al. 1993). A simple algebra shows that the corrected slope of the $f-n\left(\mathrm{H}_{2}\right)$ relation is then $\alpha(1+\beta)-\beta$ instead of $\alpha$ ( $\alpha$ is estimated, like before, supposing $\kappa_{1.3 \mathrm{~mm}}$ constant). The corrected slope is less steep when $\alpha \leq 1$, and it is positive if $\alpha$ is larger than $\beta /(1+\beta) \simeq 0.16$. The slope $\alpha$ derived previously $(0.4-0.8)$ lies well above this value of 0.16 , therefore the corrected slope is positive (i.e. the depletion factor is found to increase with density, even in the case $\kappa_{1.3 \mathrm{~mm}}$ also increases with density). We conclude that the observed variations of the depletion factor with density cannot be accounted for by variations in $\kappa_{1.3 \mathrm{~mm}}$ only. Estimates of $\mathrm{H}_{2}$ column density using the Near Infrared Color Excess method (cf. Bergin et al. 2001) that are independent of $\kappa_{1.3 \mathrm{~mm}}$ should help confirm this trend.

Assuming that a CO molecule in the gas phase has a probability equal to one to stick onto the grain after a collision (e.g. Léger 1983), the CO depletion is expected to vary roughly as $\exp (t / 8000 \mathrm{yr}) \exp \left(n\left(\mathrm{H}_{2}\right)\right)$, where $n\left(\mathrm{H}_{2}\right)$ is expressed in units of $10^{5} \mathrm{~cm}^{-3}$. We plotted in Fig. 2 this relation for $t=2 \times 10^{3} \mathrm{yr}$ and $t=2 \times 10^{4} \mathrm{yr}$ (dashed lines), as well as a "fit" for $t=8 \times 10^{3} \mathrm{yr}$ (dash-dotted line). The observed scatter between the data and the model may well reflect the difference in evolutionary stages of the prestellar cores studied here. The dash-dotted model is consistent with the results of Caselli et al. (1999) on L1544. We note that according to the models, the core ages are found relatively small compared to ambipolar diffusion timescales (Ciolek \& Basu 2001; Caselli et al. 2002b). We stress however that this simple model does not take desorption from the grains into account, and therefore the ages might be underestimated.

A more detailed study of the relation with depletion and density within the cores will be published elsewhere.

Acknowledgements. We thank the IRAM staff in Spain for assistance in the observations and the IRAM TAC for their award of observing time. A.B. was supported during this work by the Deutsche Forschungsgemeinschaft. P. André is acknowledged for his participation in the continuum observations used in this paper.

\section{References}

André, P., Ward-Thompson, D., \& Motte, F. 1996, A\&A, 314, 625

Bacmann, A., André, P., Puget, J.-L., et al. 2000, A\&A, 361, 555

Bergin, E. A., Ciardi, D. R., Lada, C. J., et al. 2001, ApJ, 557, 209

Caselli, P., Walmsley, C. M., Terzieva, R., \& Herbst, E. 1998, ApJ, 499, 234

Caselli, P., Walmlsey, C. M., Tafalla, M., et al. 1999, ApJ, 523, L165

Caselli, P., Walmlsey, C. M., Zucconi, A., et al. 2002, ApJ, 565, 331

Caselli, P., Walmlsey, C. M., Zucconi, A., et al. 2002, ApJ, 565, 344

Ciolek, G. E., \& Basu, S. 2001, ApJ, 547, 272

Evans, N. J., II, Rawlings, J. M. C., Shirley, Y. L., et al. 2001, ApJ, 557, 193

Frerking, M. A., Langer, W. D., \& Wilson, R. W. 1982, ApJ, 262,590

Fuller, G. A., \& Myers, P. C., 1993, ApJ, 418, 273

Goldsmith, P. F. 2001, ApJ, 557, 736

Goldsmith, P. F., \& Langer, W. D. 1978, ApJ, 222, 881

Henriksen, R., André, P., \& Bontemps, S. 1997, A\&A, 323, 549

Kramer, C., Alves, J., Lada, C. J., et al. 1999, A\&A, 342, 257

Ladd, E. F., Fuller, G. A., \& Deane, J. R. 1998, ApJ, 495, 871

Léger, A. 1983, A\&A, 123, 271

Penzias, A. A. 1981, ApJ, 249, 518

Preibisch, Th., Ossenkopf, V., Yorke, H. W., et al. 1993, A\&A, 279,577

Roberts, H., \& Millar, T. J. 2000, A\&A, 361, 388

Shu, F. H., Adams, F. C., \& Lizano, S. 1987, ARA\&A, 25, 23

Tafalla, M., Myers, P. C., Caselli, P., et al. 2002, ApJ, 569, 815

Ward-Thompson, D., André, P., \& Kirk, J. M. 2002, MNRAS, 329,257

Willacy, K., Langer, W. D., \& Velusamy, T. 1998, ApJ, 507, L171

Wilson, T. L., \& Rood, R. 1994, ARA\&A, 32, 191

Zucconi, A., Walmlsey, C. M., \& Galli, D. 2001, A\&A, 376, 650 